\title{
Cochrane
}

Library

Cochrane Database of Systematic Reviews

\section{End-of-life care pathways for improving outcomes in caring for the dying (Review)}

Chan RJ, Webster J, Bowers A

Chan RJ, Webster J, Bowers A.

End-of-life care pathways for improving outcomes in caring for the dying.

Cochrane Database of Systematic Reviews 2016, Issue 2. Art. No.: CD008006. DOI: 10.1002/14651858.CD008006.pub4.

www.cochranelibrary.com 
TABLE OF CONTENTS

ABSTRACT 1

PLAIN LANGUAGE SUMMARY

BACKGROUND

OBJECTIVES

METHODS

Figure 1.

RESULTS

Figure 2.

Figure 3.

DISCUSSION

AUTHORS' CONCLUSIONS

ACKNOWLEDGEMENTS

REFERENCES

CHARACTERISTICS OF STUDIES

APPENDICES

WHAT'S NEW

HISTORY

CONTRIBUTIONS OF AUTHORS

DECLARATIONS OF INTEREST

SOURCES OF SUPPORT 
[Intervention Review]

\section{End-of-life care pathways for improving outcomes in caring for the dying}

Raymond J Chan ${ }^{1}$, Joan Webster ${ }^{2}$, Alison Bowers 3

1 Institute of Health and Biomedical Innovation, Queensland University of Technology, Brisbane, Australia. ${ }^{2}$ Nursing and Midwifery Research Centre, Royal Brisbane and Women's Hospital, Herston, Australia. ${ }^{3}$ Centre for Research and Innovation, West Moreton Hospital and Health Service, Ipswich, Australia

Contact: Raymond J Chan, Institute of Health and Biomedical Innovation, Queensland University of Technology, Brisbane, Queensland, Australia.raymond.chan@qut.edu.au.

Editorial group: Cochrane Pain, Palliative and Supportive Care Group.

Publication status and date: Stable (no update expected for reasons given in 'What's new'), published in Issue 11, 2018.

Citation: Chan RJ, Webster J, Bowers A. End-of-life care pathways for improving outcomes in caring for the dying. Cochrane Database of Systematic Reviews 2016, Issue 2. Art. No.: CD008006. DOI: 10.1002/14651858.CD008006.pub4.

Copyright ( 2018 The Cochrane Collaboration. Published by John Wiley \& Sons, Ltd.

\section{A B S T R A C T}

\section{Background}

This is an updated version of a Cochrane review published in Issue 11, 2013 in the Cochrane Library. In many clinical areas, integrated care pathways are utilised as structured multidisciplinary care plans that detail essential steps in caring for patients with specific clinical problems. In particular, care pathways for the dying have been developed as a model to improve care of patients who are in the last days of life. The care pathways were designed with an aim of ensuring that the most appropriate management occurs at the most appropriate time, and that it is provided by the most appropriate health professional. Since the last update, there have been sustained concerns about the safety of implementing end-of-life care pathways, particularly in the United Kingdom (UK). Therefore, there is a significant need for clinicians and policy makers to be informed about the effects of end-of-life care pathways via a systematic review.

\section{Objectives}

To assess the effects of end-of-life care pathways, compared with usual care (no pathway) or with care guided by another end-of-life care pathway across all healthcare settings (e.g. hospitals, residential aged care facilities, community).

In particular, we aimed to assess the effects on symptom severity and quality of life of people who are dying, or those related to the care, such as families, carers and health professionals, or a combination of these.

\section{Search methods}

We searched the Cochrane Central Register of Controlled Trials (CENTRAL; Cochrane Library; 2015, Issue 6), MEDLINE, EMBASE, PsycINFO, CINAHL, review articles, trial registries and reference lists of relevant articles. We conducted the original search in September 2009, and the second updated search in July 2015.

\section{Selection criteria}

All randomised controlled trials (RCTs), quasi-randomised trials or high quality controlled before-and-after studies comparing use versus non-use of an end-of-life care pathway in caring for the dying.

\section{Data collection and analysis}

Two review authors independently assessed the results of the searches against the predetermined criteria for inclusion, assessed risk of bias, and extracted data. We used standard methodological procedures expected by Cochrane. 


\section{Main results}

We screened 3028 titles, and included one Italian cluster RCT with 16 general medicine wards (inpatient units in hospitals) and 232 carers of cancer patients in this updated review. We judged the study to be at a high risk of bias overall, mainly due to a lack of blinding and rates of attrition. Only $34 \%$ of the participants (range $14 \%$ to $75 \%$ on individual wards) were cared for in accordance with the care pathway as planned. However, these issues were to be expected due to the nature of the intervention and condition. The study population was all cancer patients in their last days of life. Participants were allocated to care using the Liverpool Care Pathway (LCP-I, Italian version of a continuous quality improvement programme of end-of-life care) or to standard care. The primary outcomes of this review were physical symptom severity, psychological symptom severity, quality of life, and any adverse effects. Physical symptom severity was assessed as overall control of pain, breathlessness, and nausea and vomiting. There was very low quality evidence of a difference in overall control of breathlessness that favoured the Liverpool Care Pathway group compared to usual care: the study reported an odds ratio (OR) of 2.0 with $95 \%$ confidence intervals (CIs) 1.1 to 3.8. Very low quality evidence of no difference was found for pain $(\mathrm{OR} 1.3,95 \% \mathrm{Cl} 0.7$ to $2.6, \mathrm{P}=0.461)$ and nausea and vomiting (OR $1.5,95 \% \mathrm{Cl} 0.7$ to $3.2, \mathrm{P}=0.252$ ). None of the other primary outcomes were assessed by the study. Limited data on advance care planning were collected by the study authors, making results for this secondary outcome unreliable. None of our other secondary outcomes were assessed by the study.

\section{Authors' conclusions}

There is limited available evidence concerning the clinical, physical, psychological or emotional effectiveness of end-of-life care pathways.

\section{PLAIN LANGUAGE SUMMARY}

\section{End-of-life care pathways for the dying}

\section{Background}

End-of-life care pathways are used for people who are in the last days of their life, to guide effective care and aid decision making. Due to substantial concerns regarding safety and quality of care associated with the pathway implementation, the most used end-of-life care pathway (Liverpool Care Pathway) is no longer used in the United Kingdom (UK). This review examined whether using end-of-life care pathways in caring for the dying was effective.

\section{Study characteristics}

In July 2015, we searched scientific databases for clinical trials in which the effect of the end-of-life care pathway was compared with a control group that received usual care, or with trials comparing one end-of-life care pathway with another end-of-life care pathway. Participants were patients, carers and families who received care guided by an end-of-life care pathway. There were no restrictions on age of the patient, diagnosis or setting (hospital, home, nursing home).

\section{Key results}

In the current review we found one Italian study, in which information about 232 patients who were dying was provided by their informal carers (friends or family). Only $34 \%$ of the participants were cared for in accordance with the pathway. Breathlessness was better controlled for patients on the Liverpool Care Pathway compared to patients not on the pathway, but this is based on evidence from one small trial. The study did not report on important outcomes such as the severity of other physical or psychological symptoms or quality of life, or if there were any side effects associated with using the end-of-life care pathway. Nor were there questions about satisfaction with care, costs of the intervention, or quality of communication between carers and healthcare providers.

\section{Quality of evidence}

We judged the included study to be of very low quality due to potential biases, including: not being able to prevent participants from knowing which group they were in (usual care or the care pathway group); the large number of carers who were initially enrolled, but who did not respond to follow-up questionnaires (this was particularly true for carers of patients in the wards where the care pathway was not used (control wards)); the low proportion of patients who actually received the care pathway (intervention) as planned; and that the study only included cancer patients in Italian hospitals; therefore, results might not apply to patients with other diseases. 


\section{B A C K G R O U N D}

This is an updated version of a Cochrane review published in Issue 11, 2013 in the Cochrane Library.

\section{Description of the condition}

It is well recognised that populations in developed countries are ageing (United Nations 2013). As populations age, the pattern of diseases from which people die also changes (WHO 2011). With advanced ageing, there is an increased risk of death from chronic diseases, such as cancer and heart failure. For example, cancer was estimated to account for 7.6 million deaths ( $12 \%$ of all deaths) worldwide in 2008 (WHO and Cancer Research UK 2012). Therefore, palliative care has been identified as one of the worldwide public health priorities due to the ageing population (WHO 2011). While palliative care is concerned with "the quality of life of patients and families who face life-threatening illness, by providing pain and symptom relief, spiritual and psychosocial support from diagnosis to the end of life and bereavement" (WHO 2009), end-of-life care focuses on the last days and hours of life (Lunney 2003). The need to provide high quality end-of-life care is essential. The needs of dying people may include, but are not limited to, knowing when death is coming, understanding what can be expected, being able to maintain a sense of control and having their wishes given preference, having access to information and excellent care, and having access to spiritual and emotional support as required (Steinhauser 2000; Steinhauser 2001). Quality end-of-life care may vary from person to person and may be difficult to define and measure accurately. However, such care should at least include the following domains: quality of life, physical symptoms, emotional and cognitive symptoms, advance care planning, functional status, spirituality, grief and bereavement, satisfaction and quality of care, as well as carer's well-being (Mularski 2007).

Obstacles to quality end-of-life care have also been identified and may include failure to recognise treatment futility, lack of communication among decision makers, no agreement on a course of end-of-life care, and failure to implement a timely end-oflife plan of care (Travis 2002). In recent years, there has been a variety of initiatives developed worldwide to target such issues by developing systemic approaches towards end-of-life care. These initiatives include programmes such as the National End of Life Care Programme (Department of Health 2008), Gold Standards Framework in Care Homes (Badger 2007), and the Liverpool Care Pathway (Ellershaw 1997; Ellershaw 2003a).

\section{Description of the intervention}

Integrated care pathways are documents that outline the essential steps of multidisciplinary care in addressing a specific clinical problem. They can be used to introduce clinical guidelines and systematic audits of clinical practice (Hockley 2005). The Liverpool Care Pathway is an example of an integrated care pathway specifically for the dying phase of palliation.

Historically, dying patients receiving general hospital care tended to lack adequate attention from senior medical and nursing staff (Mills 1994). The quality of symptom control and basic nursing care were considered to be inadequate (Mills 1994). It was thought that much could be learned from the way patients were cared for in the hospice movement (Mills 1994). The Liverpool Care Pathway was an example of strategies developed by the Royal Liverpool University
Trust (UK) and the Marie Curie Centre Liverpool (UK) (Ellershaw 1997; Ellershaw 2003a), based on the care received by people in the hospice setting. Other objectives of the pathway were to promote cost-effective health care by appropriate prescribing, and avoiding crisis interventions and inappropriate hospital admissions. The document is patient-centred and focuses on the holistic needs of people who are dying. It incorporates the physical, psychological, social, spiritual and religious aspects of care (Ellershaw 2007). The Liverpool Care Pathway defines 19 goals considered essential in the management of dying patients, and for the care of their relatives/ carers after death (Ellershaw 1997; Ellershaw 2003a). These goals were established with the issues identified from surveys, focus groups, expert opinion and consensus best practice.

Later, several other groups developed care pathways for dying people based on the concept of Ellershaw and colleagues (Bookbinder 2005; Fowell 2002; Pooler 2003). While the professional conjecture is that end-of-life care pathways promote best possible patient outcomes (Ellershaw 2007), speculation has suggested possible adverse effects. These controversies included premature use of the pathway, leading to death due to the premature diagnosis of imminent death, the care pathway masking the signs of improvement in patients and causing carers' dissatisfaction (Delvin 2009; Smith 2009). There have been substantial concerns raised by the public and by health professionals that have been documented in the UK Governmentcommissioned, independent review of the Liverpool Care Pathway led by Baroness Julia Neuberger (Neuberger 2013). The panel reviewed multiple sources including: written submissions from the public and health professionals with experience of the Liverpool Care Pathway, the academic literature and hospital complaints. The panel concluded that the Liverpool Care Pathway, used generically for all patients in the last hours or days of life, was the wrong approach (Neuberger 2013). The report also highlighted the complexity around the use of the pathway, specifically highlighting a number of ethical, safety, clinical practice and negligence issues and how inadequately dying is diagnosed in clinical care (Neuberger 2013). As a result of the review in July 2013, the UK government made a decision to phase out the pathway nationally over the six to 12 months after the release date of the Neuberger 2013 review.

It is particularly important to recognise that an end-of-life care pathway is a complex intervention (McConnell 2013; Medical Research Council 2000). Although there are methodological considerations and challenges, it is still important to conduct as rigorous an evaluation as possible to determine the effects (benefits or harms) of the end-of-life care pathways (Chan 2014; Currow 2011; Medical Research Council 2000). Therefore, a systematic review is warranted to substantiate claims as to whether the end-of-life care pathways are beneficial or harmful for dying patients and their carers.

\section{How the intervention might work}

In many clinical areas, integrated care pathways are utilised as structured multidisciplinary care plans that detail essential steps in caring for patients with specific clinical problems (Campbell 1998). Care pathways for the dying have been developed as a model to improve the end-of-life care of all patients. They ensure that the most appropriate management occurs at the most appropriate time, and that it is provided by the most appropriate health professional. 


\section{Why it is important to do this review}

Two systematic reviews reported that clinical pathways enhance efficiency of care, without adverse effects on outcomes, among patients who undergo gastrointestinal surgery (Lemmens 2008), and show a significant length of stay reduction in patients who undergo invasive procedures (Rotter 2008). Both of these systematic reviews included evidence involving designs such as RCTs and other types of controlled studies.

In contrast, one Cochrane systematic review reported that there was no significant benefit in functional outcome and patient satisfaction, and that quality of life might actually be made worse for patients following stroke care pathways (Kawn 2004). Therefore, clinical pathways seem to be beneficial for managing certain clinical problems, but not all.

Over recent years, clinical pathways for end-of-life care management have been used widely around the world and have been established as a part of the end-of-life care policies or strategies in some countries (Department of Health and Ageing 2011; Ministry of Health NZ 2013). A variety of end-of-life care pathways have been used in Australia, the Netherlands, United Kingdom, China, Ireland and United States (Phillips 2011). There is a significant need for clinicians to be informed about the utilisation of end-of-life care pathways with a systematic review.

\section{OB JECTIVES}

To assess the effects of end-of-life care pathways, compared with usual care (no pathway) or with care guided by another end-oflife care pathway across all healthcare settings (e.g. hospitals, residential aged care facilities, community).

In particular, we aimed to assess the effects on symptom severity and quality of life of people who are dying, or those related to the care, such as families, carers and health professionals, or a combination of these.

\section{METHODS}

\section{Criteria for considering studies for this review}

\section{Types of studies}

We included clinical trials in which the effect of the end-of-life care pathway could be compared with a control group that received usual care, or with trials comparing one end-of-life care pathway with another end-of-life care pathway. We planned to include RCTs, cluster RCTs and quasi-RCTs.

If limited RCTs and quasi-RCTs were available, we planned to consider including controlled before-and-after studies. The review authors adopted the criteria for inclusion of controlled before-and-after studies from the Cochrane Effective Practice and Organisation of Care Review Group guidelines (EPOC 2002). These criteria include: (1) contemporaneous data collection, (2) appropriate choice of control site, and (3) a minimum of two intervention sites and two control sites. We did not plan to include any non-controlled studies (EPOC 2002). The analysis for randomised and non-randomised studies were to have been undertaken separately because non-randomised comparisons may overestimate treatment effects (Chalmers 1983; Sacks 1982), and the size and direction of the bias can be unpredictable (Deeks 2003).

\section{Types of participants}

We included patients and carers/families who had received care guided by an end-of-life care pathway. We included participants with different diseases such as cancer. However, participants who received interventions must have been receiving care guided by an end-of-life care pathway for their last days and hours of life. We applied no restrictions on age of the patient, diagnosis or setting (hospital, home, nursing home).

\section{Types of interventions}

The planned comparisons were:

- intervention (receiving care guided by an end-of-life care pathway) versus usual care;

- intervention A (pathway A) versus intervention B (pathway B).

An end-of-life care pathway may have been part of a larger intervention; we only included these studies if the effect of the pathway could be isolated.

\section{Types of outcome measures}

\section{Primary outcomes}

- Physical symptom severity (measured by any instrument used by the study author such as Edmonton Symptom Assessment Scale (Bruera 1991)), Memorial Symptom Assessment Scale (Portenoy 1994).

- Psychological symptom severity (measured by any instrument used by the study author. For example, Hospital Anxiety and Depression Scale (Zigmond 1983)).

- Quality of life (measured by any instrument used by the study author such as McGill Quality of Life Questionnaire (Cohen 1995)).

- Harms (any adverse effects, as determined by the researchers, health professionals or carers/families).

\section{Secondary outcomes}

- Advance care planning (as measured by whether advance care planning had happened or not).

- Communication between healthcare teams and families (as measured by the occurrence of any family meetings).

- Carers' well-being.

- Grief and bereavement.

- Patient/staff/carers' satisfaction.

- Staff confidence.

- Cost of intervention.

- Cost of care.

- Medication/treatment use.

- Spiritual needs.

We included any tools used by the study authors of the included studies.

\section{Search methods for identification of studies}

The search was run for the original review in September 2009 and the subsequent search was run in June 2013 (the first update). This second update of the review includes the search last conducted in July 2015. 


\section{Electronic searches}

For this update we searched:

- the Cochrane Central Register of Controlled Trials (CENTRAL; Cochrane Library; 2015, Issue 6 of 12);

- MEDLINE (OVID) June 2013 to 27 July 2015;

- EMBASE (OVID) June 2013 to 27 July 2015;

- PsycINFO (OVID) 2013 to 27 July 2015;

- CINAHL (EBSCO) June 2013 to 27 July 2015.

We developed the search strategy to comprise searches for both keywords and medical subject headings under existing database organisational schemes. The strategies for each database are presented in Appendix 1, Appendix 2, Appendix 3, Appendix 4 and Appendix 5. We applied no restrictions by language. We translated foreign language abstracts for the application of the inclusion and exclusion criteria, and, where necessary, we planned to translate the methods, results and discussion sections for inclusion in the review.

\section{Searching other resources}

We searched the reference lists of any relevant reviews or other studies, scanned paper issues of journals relevant to interventions of end-of-life care pathway and scanned abstracts from relevant conference proceedings. We also used Google to search the World Wide Web, Caresearch (www.caresearch.com.au), and the ProQuest Dissertations and Theses database for grey literature and conference abstracts (www.proquest.com). We searched databases in TrialsCentral (www.trialscentral.org), the World Health Organization's (WHO) Clinical Trial Search Portal (www.who.int/trialsearch), and Current Controlled Trials (www.controlled-trials.com), to identify ongoing or recently completed studies. We planned, if applicable, to present relevant ongoing studies in a table in the review.

\section{Data collection and analysis}

\section{Selection of studies}

Two review authors (RC, $A B$ ) prescreened all search results (titles and abstracts) for possible inclusion, and those selected by either or both review authors were subject to full-text assessment. Two review authors independently assessed the selected articles for inclusion. We resolved any discrepancies by consensus, overseen by a third review author acting as arbiter, with approval by one review author and the arbiter being sufficient (see Figure 1 ). We also listed those studies excluded after full-text assessment in the table Characteristics of excluded studies, giving reasons for exclusion. 
Figure 1. Study flow diagram.

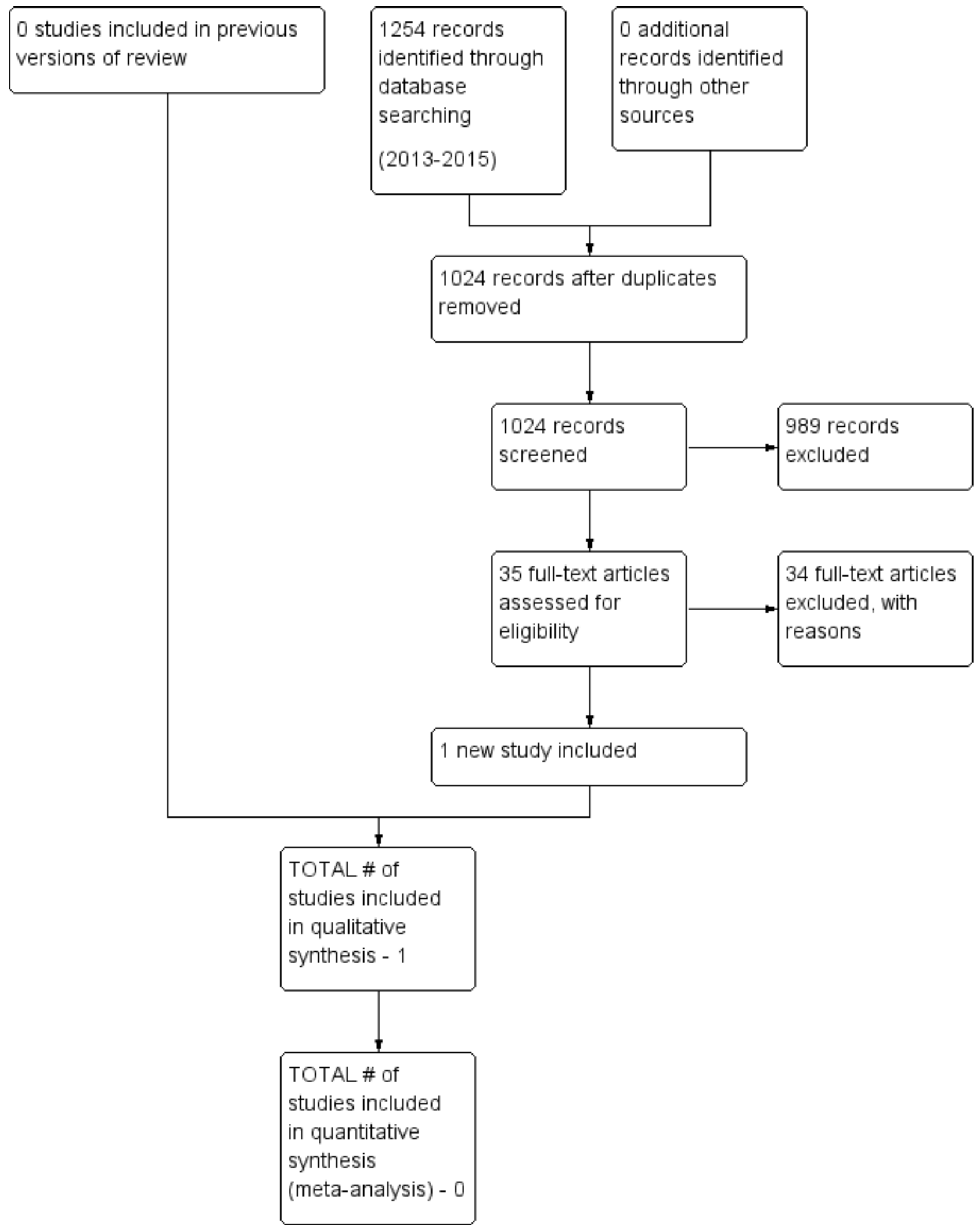




\section{Data extraction and management}

We developed a data extraction form based on the Cochrane Pain, Palliative and Supportive Care Review Group's template. We extracted the following main sets of data from each included study:

- lead author;

- date;

- study participant inclusion criteria;

- participants (participant diagnoses/condition(s) and demographics: race/ethnicity, gender, religion/culture, socioeconomic status, age);

- study design and timetable, randomisation, allocation concealment;

- interventions (end-of-life care pathway type);

- intervention setting (hospital, home, residential aged care facilities);

- numbers of participants in each trial arm, withdrawals and dropouts;

- outcome measures; time(s) at which outcomes were assessed.

Two review authors independently extracted data on to the data extraction form. Any discrepancies would have been referred to a third review author and any errors or inconsistencies resolved.

\section{Assessment of risk of bias in included studies}

We assessed and reported on the risk of bias of the included study in accordance with the guidelines in the Cochrane Handbook for Systematic Reviews of Interventions (Higgins 2011), which recommends the explicit reporting of the following individual domains.

\section{The criteria for RCTs were:}

- sequence generation;

- allocation concealment;

- blinding of participants and personnel

- blinding of outcome assessors (assessed for each main outcome or class of outcome);

- incomplete outcome data (assessed for each main outcome or class of outcome);

- selective outcome reporting;

- other sources of bias.

The criteria for controlled before-and-after studies were:

- baseline measurement of outcomes;

- baseline characteristics of studies using second site as control;

- protection against exclusion or selection bias;

- protection against contamination;

- reliable primary outcomes measures;

- appropriate analysis of data.

We have examined and reported the following:

- validation and reliability of outcome measures;

- whether the study obtained ethics committee approval and ensured informed consent for participation;
- use of standardised protocols for information delivery. We were to have checked for consistency of the delivery of interventions where possible.

We classed studies as at overall high risk of bias if one of the following domains was deemed to be at high risk of bias: generation of randomisation sequence, allocation concealment, blinded outcome assessment. We have presented our assessment in 'Risk of bias' tables for the included study. We contacted study authors for additional information about the study methods as necessary. We have incorporated the results of the risk of bias assessment into the review through narrative description and commentary about each of the items mentioned.

\section{Measures of treatment effect}

For individual studies, effect measures for categorical outcomes were to include risk ratio (RR) or odds ratio (OR) with their $95 \%$ confidence intervals (Cls). For statistically significant effects, we would have calculated number needed to treat for an additional beneficial effect (NNTB). If possible, for continuous outcomes, the effect measure was to have been mean difference (MD) or, if the scale of measurement differed across trials, standardised mean difference (SMD), each with its $95 \% \mathrm{Cl}$. For meta-analyses (see below), for categorical outcomes, we would have calculated typical estimates of RR with their $95 \% \mathrm{Cl}$; and for continuous outcomes, we would have calculated the MD or a summary estimate for SMD, each with its $95 \% \mathrm{Cl}$.

We would have analysed data using Cochrane's Review Manager 5 software (RevMan 2014).

\section{Unit of analysis issues}

We did not expect to find cross-over trials for this type of intervention due to the end-of-life pathway nature. We had planned that if cluster randomised trials had been identified, we would have reported the intracluster correlation coefficient and adjusted for clustering if possible. In this review, the authors of the only included study had adjusted for clustering, so results are presented as reported in the trial paper.

\section{Dealing with missing data}

If some outcome data remained missing despite our attempts to obtain complete outcome data from authors, we would have performed an available-case analysis, based on the numbers of participants for whom outcome data were known. If standard deviations (SDs) were missing, we would have imputed them from other studies, or where possible, computed them from standard errors (SEs) using the formula SD $=\mathrm{SE} \times \sqrt{ }^{-} \mathrm{N}$, where these were available (Higgins 2011). We also planned to report on levels of drop-outs in the intervention and comparison groups as an indicator of 'acceptability' of the intervention, and the likelihood of bias.

\section{Assessment of heterogeneity}

We would have tested heterogeneity using the $\mathrm{Chi}^{2}$ statistic and any heterogeneity was to have been further quantified with the 12 statistic (which describes the percentage of the variability in effect estimates that is due to heterogeneity rather than sampling error). We would have considered a value greater than $50 \%$ as representing substantial heterogeneity (Higgins 2011). 


\section{Assessment of reporting biases}

We would have assessed reporting bias using guidelines in the Cochrane Handbook for Systematic Reviews of Interventions (Higgins 2011). We did not expect to find a large number of studies, so we thought it was unlikely that publication or inclusion bias would be assessed. However, we planned to do a funnel plot if enough studies were available to do a meaningful assessment of publication bias.

\section{Data synthesis}

If studies had been sufficiently similar in terms of population, inclusion criteria, interventions, outcomes (including the time(s) at which these are assessed), or a combination of these, we would have considered pooling the data statistically using metaanalysis. We would have reported the results of the individual trials separately where the outcome data were unsuitable for meta-analysis. We planned to use fixed-effect models when population measures were similar and random-effects models where population parameters varied from study to study.

\section{Subgroup analysis and investigation of heterogeneity}

We would have conducted subgroup analyses if sufficient data could support the analyses. Subgroups may have included disease types and settings where care was received.

\section{Sensitivity analysis}

If there were other sources of heterogeneity, we planned to explore further by using sensitivity analysis to determine the effects of the end-of-life care pathways, overall methodological quality and use of intention-to-treat analysis. We would have removed studies with high attrition rates (over 50\%) from the meta-analysis to determine whether the results would be significantly different without them.

\section{RE S U L T S}

\section{Description of studies}

See: 'Characteristics of included studies'; 'Characteristics of excluded studies'; 'Characteristics of ongoing studies'.

\section{Results of the search}

For this update, we found and assessed 1024 titles and abstracts in electronic format after we had removed duplicates. Of the 1024 titles and abstracts we screened, we assessed 35 as relevant, and one we included one study (Costantini 2014): see Figure 1 (the PRISMA study flow chart). We identified one ongoing study (see Characteristics of ongoing studies).

\section{Included studies}

We included one cluster RCT in this review (Costantini 2014): see Characteristics of included studies for more details. The study included 16 Italian hospital wards. Data were retrieved for 308 patients who died from cancer; 232 family members were interviewed for outcome assessment.

\section{Intervention}

\section{Description of the intervention}

The intervention in the Costantini 2014 trial involved the implementation of an Italian version of the Liverpool Care Pathway (LCP-I), a continuous quality improvement programme of end of-life care. The LCP-I is a complex 10-step intervention which involves: development of the project in the targeted setting (three steps); implementing the project over a six-month period (five steps) and development of a sustainability strategy (two steps). The LCP-I was implemented by a specialist palliative care team in eight Italian hospital medical wards that had been paired and randomly allocated to receive the LCP-I intervention or standard healthcare practice (control arm) (Costantini 2014). All eight wards that were allocated to the LCP-I intervention commenced the intervention, however three hospital wards stopped using the LCPI documentation at the end of the experimental phase. Fifty-one participants $(34 \%)$ were cared for in accordance with the LCP-I programme, with proportions in eight hospital wards ranging from $14 \%$ to $75 \%$. The median time on LCP-I in the eight hospitals was 31.5 hours (range 4 to 57 ).

The LCP-I programme includes 10 steps:

\section{Development of the implementation project}

Step 1: Establishment of the project and preparation of the environment

- Identify and describe the characteristics of the ward

- Identify and describe the characteristics of the palliative care team (PCT)

- Obtain consent from hospital management and the head of the ward

- Present the general outlines of the LCP-I programme to the ward staff

- Outline the LCP-I programme on the ward

- Begin the approval procedure for the training programme

- Register the project at the national centre for LCP-I

Step 2: Development of the documentation

- Acquire educational materials for training

- Prepare the necessary documentation for the ward

Step 3: Base review-retrospective evaluation of variances

- Review the medical documentation of the patients who died in the ward

- Investigate the variances with the ward staff

Implementation of the LCP-I programme (6.0 months)

Step 4: Intensive education programme ( $\leq 1$ month)

- Undertake three modules of 4 hours (total 12 hours), repeated twice to allow the participation of all clinical ward staff (doctors and nurses)

Step 5: Clinical implementation of the LCP-I documentation intensive support to the ward staff (1.5 months)

- The ward staff, closely supported by the PCT team, oversee the implementation process, and start using the LCP-I documentation for patients who are dying

Step 6: Semi-intensive support to the ward staff (1.5 months)

- Ward staff, accompanied by the PCT team overseeing the implementation process, learn to use the LCP-I documentation 
as a standard procedure for patients who are dying; clinical audits are planned for difficult cases

Step 7: Assessment and further training (at the end of the fourth month)

- PCT team assesses the outcome of the preliminary steps with the aim of developing an appropriate training strategy for the ward staff during the subsequent stages of the implementation process

Step 8: Consolidation phase (2.0 months)

- LCP-I documentation is established in the ward as an indicator of the quality of end-of-life care for all patients who are dying; the PCT team support the ward staff using the most suitable means for consolidation of the changes introduced by the LCPI programme

\section{Sustainability of high standards of quality of end-of-life care}

Step 9: Initiation of a strategy for sustainability

- The LCP-I programme is established as a routine procedure on the ward and in the hospital

- Develop an end-of-life care strategy for the ward

Step 10: Regional and national strategy

- Use the outcome of the trial study to stimulate discussions at regional and national levels about issues linked to the quality of end-of-life care

\section{Participants}

On an individual level, the trial involved all cancer patients who died from cancer with a classification of 140.0 to 239.9 , as listed by the International Classification of Diseases Ninth Revision (Centers for Disease Control and Prevention 2016). The patient's closest family member, as identified during the patient's last week of life as the patient's main caregiver, was the individual contacted to participate in the interview. The interview with the caregiver had to take place between two and four months after the patient's death and be conducted face-to-face, unless there were exceptional cases where it was impracticable for the caregiver to attend in person; in this circumstance the interview was to be conducted by telephone (Costantini 2014).

\section{Outcomes}

Costantini 2014 assessed outcomes in the six months after the end of the LCP-I intervention, during face-to-face interviews with the patient's family member identified as the main caregiver. Questions from the Global Scale of the tool kit "After-death Bereaved Family Member Interview" (Teno 2001) and the Italian version of the "View of informal carers-evaluation of service" (Costantini 2005) were used in the interviews. The seven scales were informing and making decisions; advance care planning; respect, dignity and kindness; family emotional support; co-ordination of care; family self efficiency and overall rating of patient-focused, family-centred care. The scores of each scale were calculated using a scale of $0-100$, with a score of 0 indicating worst end-of-life care and 100 best endof-life care.

\section{Excluded studies}

We excluded 28 studies in the original review, 32 (an additional four) in the first update, and 34 (an additional two) in this current update because the study designs did not meet the criteria for included studies. We excluded three controlled before-and-after studies because they did not meet the minimum criteria to be included in this review. These criteria include: (1) contemporaneous data collection, (2) appropriate choice of control site, and (3) a minimum of two intervention sites and two control sites (EPOC 2002).

\section{Risk of bias in included studies}

We deemed the included study to be at high risk of bias overall (Figure 2 and Figure 3). 
Figure 2. Risk of bias summary: review authors' judgements about each risk of bias item for each included study.

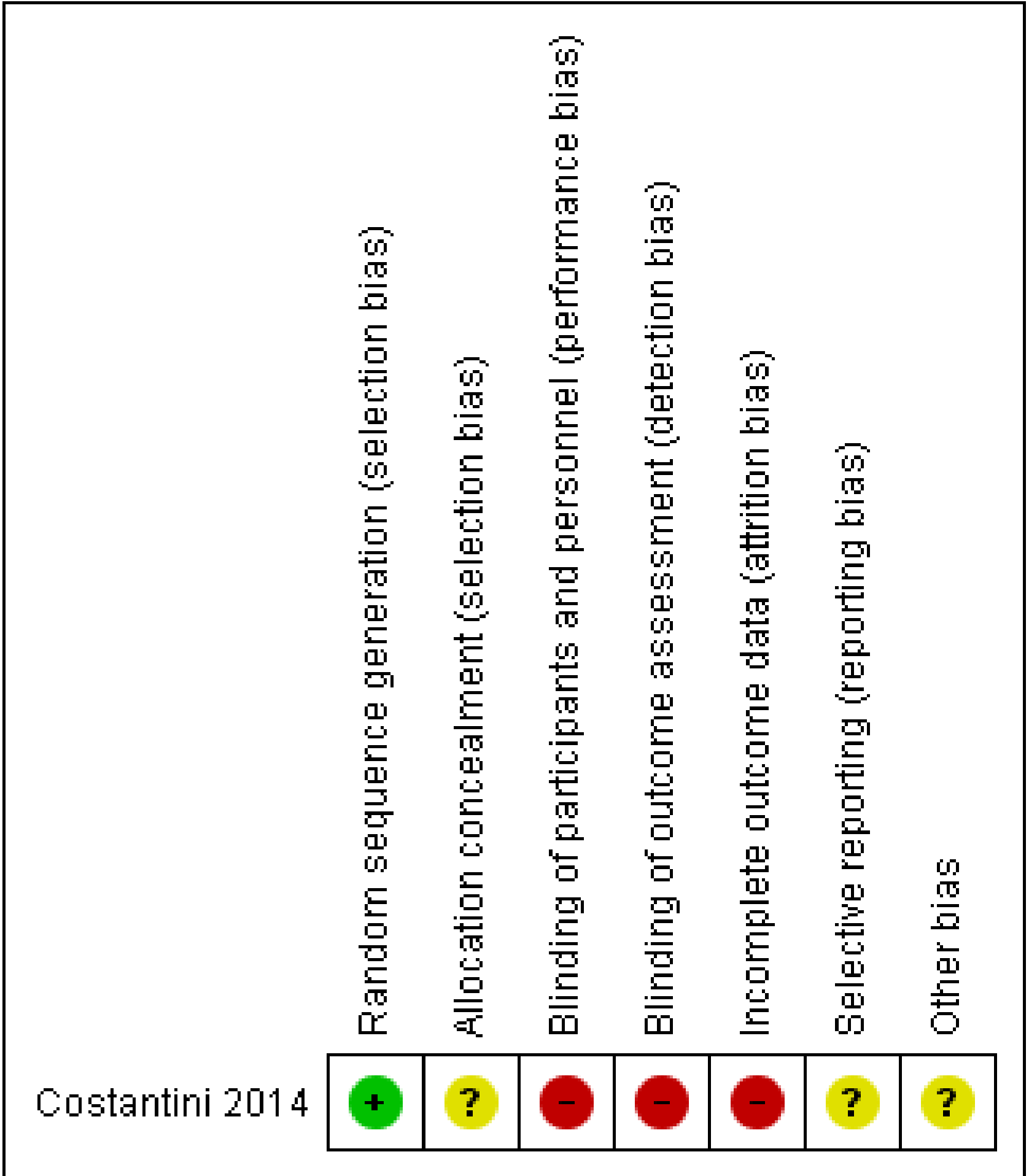


Figure 3. Risk of bias graph: review authors' judgements about each risk of bias item presented as percentages across all included studies.

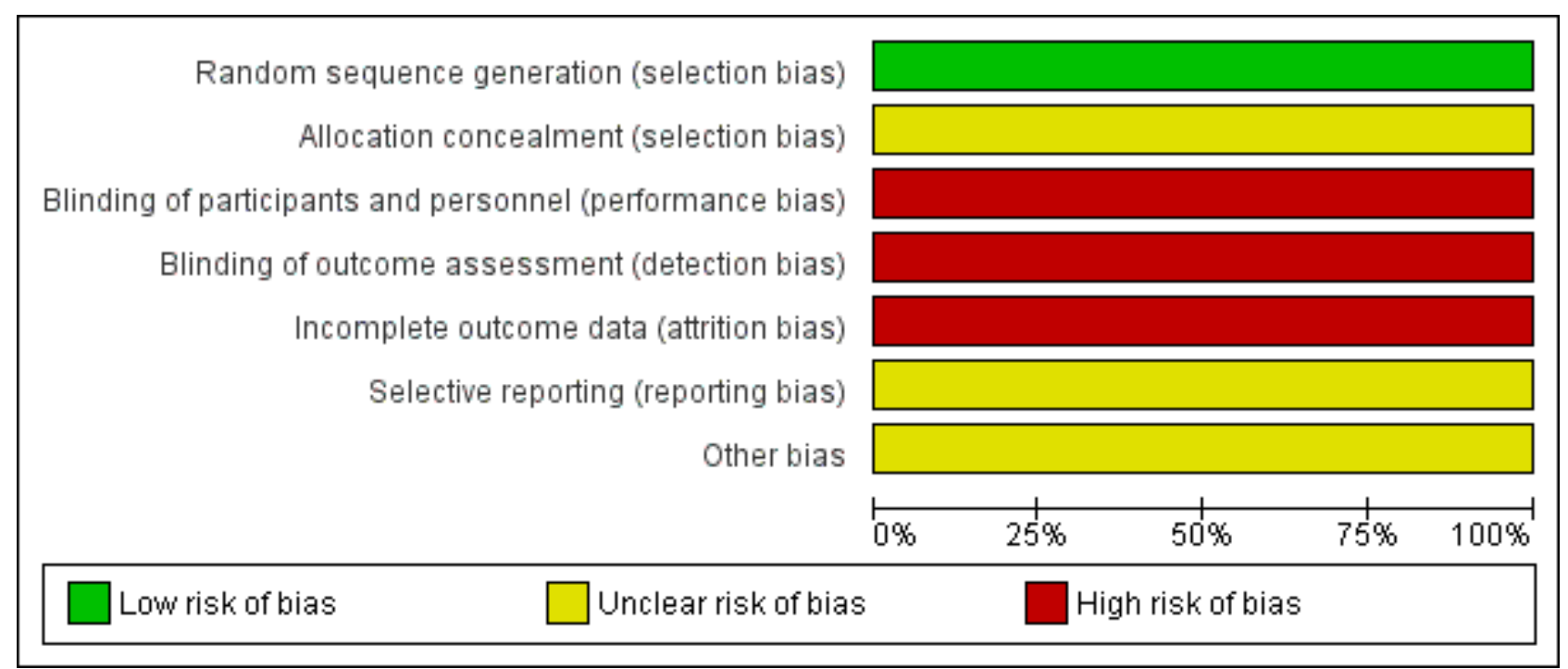

\section{Allocation}

We classed the Costantini 2014 trial as being at low risk of bias for generation of random sequence. The trial centre of the National Cancer Research Institute of Genoa, randomised and verified the eligibility of wards, recorded details of each pair of wards and matched PCTs, assigned a numerical code for identification, and recorded the allocation (Costantini 2014).

In terms of allocation concealment, we classed the study as being at unclear risk of bias as no information regarding allocation of the random sequence was reported.

\section{Blinding}

We classed the trial as being at high risk of bias as none of the hospital staff, PCTs, or interviewers were blinded to the intervention. There was no indication that they conducted a blinded outcome assessment (Costantini 2014).

\section{Incomplete outcome data}

We considered the study to be at high risk of bias for this domain Only $232(75 \%)$ of 308 family members enrolled were interviewed. In addition, a higher numbers of participants were lost to follow-up in the control arm: $119(81 \%)$ of 147 with relatives cared for in the LCP-I wards and $113(70 \%)$ of 161 in the control wards (Costantini 2014).

\section{Selective reporting}

We deemed reporting bias to be an unclear risk of bias, as although the authors appear to have reported all that they planned to measure, trial registration was first received "March 4, 2010" (Costantini 2014) by ClinicalTrials.gov, which was four months after the trial commenced.

\section{Other potential sources of bias}

add text here

\section{Effects of interventions}

\section{Primary Outcomes}

\section{Physical Symptom Severity}

The Costantini 2014 trial reported the patients on the LCP-I wards, as rated by the caregivers, had overall better control of breathlessness (OR 2.0, 95\% $\mathrm{Cl} 1.1$ to $3.8, \mathrm{P}=0.026$ ). However, there were no statistically significant differences in overall control of 'pain' (OR $1.3,95 \% \mathrm{Cl} 0.7$ to $2.6, \mathrm{P}=0.461$ ) and 'nausea or vomiting' (OR $1.5,95 \% \mathrm{Cl} 0.7$ to $3.2, \mathrm{P}=0.252$ ) between those on the LCP-I wards and control wards.

The Costantini 2014 trial did not measure the other primary outcomes of this review, such as psychological symptom severity, quality of life or harms/adverse effects.

\section{Secondary Outcomes}

The Costantini 2014 trial measured advance care planning, however, no meaningful results for this outcome were reported due to the small sample size.

None of our other predefined secondary outcomes, such as communication between healthcare teams and families (as measured by the occurrence of any family meetings), carers' wellbeing, grief and bereavement, patient/staff/carers' satisfaction, staff confidence, cost of intervention, cost of care, medication/ treatment use, and spiritual needs were assessed.

\section{DISCUSSION}

\section{Summary of main results}

Of the 1024 titles screened, we included one study with a total of 232 responses from care providers of patients who were in their last days of life (Costantini 2014). The study, a cluster RCT of 16 hospital wards, compared the Liverpool Care Pathway (LCP) with standard care. Most of the primary and secondary outcomes for this review were not assessed in the included trial. The physical symptoms assessed were 'control over 'breathlessness', 'pain' and 
'nausea and vomiting'. The only difference, favouring the Italian version of the Liverpool Care Pathway (LPC-I) group, was control over breathlessness.

\section{Overall completeness and applicability of evidence}

The effectiveness of end-of-life care pathways has only been evaluated in one study that evaluated the use of the LCP in one country and among a population of cancer patients. Thus, results cannot be generalised beyond this population, using this particular pathway, in a hospital setting.

\section{Quality of the evidence}

We judged the study to be at a high risk of bias overall. The study was small and therefore statistically under-powered to detect treatment differences should they exist. Confidence intervals reported in the paper were wide for all outcomes, indicating imprecision in effect estimates. We judged the study to be at high or unknown risk of bias across all domains except randomisation. Outcome evaluation was limited by the low response rate from carers, particularly in the control group. A low adherence to the study protocol was also a limitation of the study. The trial report would have been improved if the good practice conduct and reporting guidelines, e.g. CONSORT (Schultz 2010) had been followed. We downgraded the quality of evidence to very low for the reasons stated above, and because the data were derived from a single study with a small number of participants.

\section{Potential biases in the review process}

We used an extensive search strategy to identify relevant studies, based on previous Cochrane reviews and on other reviews with different strategies. It is unlikely that we have overlooked relevant high quality large studies of end-of-life care pathways, especially because the end-of-life care pathways have received much attention in the press and in academic editorials (Chan 2014; Chan 2014b; Currow 2014).

\section{Agreements and disagreements with other studies or reviews}

A qualitative substudy of the Costantini 2014 trial was conducted, which explored the views of six nurses and five physicians who were involved with the implementation of the LCP-I (Di Leo 2013). Issues raised in the thematic analysis were similar to the concerns and issues raised in the Neuberger 2013 review; in particular, concerns over the actual implementation of the LCP-I and its interpretation. Key concerns and issues raised by respondents of the Di Leo 2015 study included:

- the varying levels of staff competency due to the irregular level of participation in the education programme;

- the limitations of the LCP-I documentation as the LCP-I did not take into account the patients' specific therapies or clinical situation;

- the correct point in time when the LCP-I should be implemented;

- the inappropriateness of the hospital ward environment due to the issue that individuals with very different care needs were admitted to the same room as individuals on the LCP-I (with lack of privacy being a major concern);
- Lack of the dedicated time required to implement the LCP-I fully due to the busy nature and workload of a hospital ward compared to that of a hospice;

- the difficulty recognising and responding to patients' needs due to a difference in the aim of the LCP-I and a general medical ward, namely the preservation of life regardless of clinical condition; and

- the fact that the LCP-I did not account for the expectation of family members who required an 'action-orientated' approach to care for their family member.

Although the UK government has made a decision to phase out the LCP (the most commonly used end-of-life care pathway) in the UK, such decisions have not been made in other countries. With a number of safety concerns reported by Neuberger's report and the lack of evidence (Neuberger 2013), it is extremely difficult to determine whether the suggested negative consequences associated with the LCP are associated with the actual pathwaybased care; poor implementation of pathway-based care; the emotional consequences of illness, death and bereavement; or a combination of these (Parry 2013). In addition to the Costantini 2014 trial, there is an urgent need for rigorous research to answer several key questions: do the adverse findings from Neuberger's report apply to countries other than the UK and, if so, to what extent? What are the differences in outcomes between the LCP and other end-of-life care pathways? Are the outcomes for dying patients who are placed on an end-of-life care pathway different to those receiving usual care? If the answers to these questions are 'yes': are the right people put on the end-of-life care pathway at the right time in their illness trajectory? In which settings should an end-of-life care pathway be used? How senior should the clinicians be and how much history of this patient should they have before an end-of-life care pathway is initiated?

The results of a number of case series and non-eligible controlled or non-controlled before-and-after studies indicated that end-oflife care pathways may have the potential to improve symptom management (Bailey 2005; Veerbeek 2008); clinical documentation and assessment (Bookbinder 2005; Luhrs 2005; Veerbeek 2008); knowledge of end-of-life care among internal medicine students (Okon 2004); prescription of medications for end-of-life (Bailey 2005; Mirando 2005); bereavement levels of relatives (Veerbeek 2008a); and outcomes in relation to respect, kindness, dignity, family emotional support, family self efficacy and co-ordination of care (Costantini 2013a). However, the effects of pathways are difficult to ascertain from these designs. Moreover, none of the excluded studies reported the adverse effects of any end-of-life care pathway.

Designing and conducting trials involving the dying is difficult and challenging due to methodological and ethical issues (Fowell 2004; Karlawish 2003). These issues may include difficult patient recruitment due to the patient being too ill to participate or unable to give informed consent, or the heterogeneous nature of palliative populations (Addington-Hall 2007). However, researchers should attempt to investigate end-of-life interventions with the most rigorous research methodology possible. A range of other strategies can also be considered to make clinical trials possible. These include designing a shorter term study, limiting the number of outcomes, undertaking frequent follow-ups, advance consent and proxy consent where appropriate for studies involving this population (Reyna 2008). Although the challenges in conducting 
clinical research for the dying are well recognised, the Costantini 2014 team acknowledged the importance of generating high quality evidence to inform practice in this area and completed a phase III cluster RCT to test the effectiveness of the LCP.

\section{AUTHORS' CONCLUSIONS}

\section{Implications for practice}

\section{For people receiving end-of-life care and families}

Although end-of-life care pathways are generally developed with best practice principles, the only randomised controlled trial (RCT) available did not demonstrate important benefits for patients and their families/caregivers.

\section{For clinicians}

Although strong evidence supporting end-of-life care pathways is lacking, the principles underpinning such pathways remain relevant. Plans for end-of-life care should be developed in open consultation with the patient and significant others.

\section{For policy makers and funders}

Without strong evidence supporting the effectiveness of the endof-life care pathways, it is difficult to justify the implementation of an end-of-life care pathway given the resource intensive nature of the intervention. It would be prudent to invest resources into other evidence-based strategies for improving the standards of end-of-life care. All health services using an end-of-life care pathway are encouraged to have their use of the pathway, to date, independently audited, with particular emphasis on the Neuberger's findings (Neuberger 2013). Any subsequent use should be based on carefully documented prospective evaluations.

\section{Implications for research}

\section{General}

Although high level evidence remains scarce, existing research may be used to inform the development of any future trials. The only available RCT failed to show any significant benefits.

\section{Design}

There remains an urgent need for large RCTs or other well-designed controlled studies for the evaluation of the use of end-of-life care pathways in caring for dying people in various settings.
Such evaluations might be difficult in countries where end-of-life care pathways are embedded in practice or are being withdrawn. However, it remains important to test the effectiveness of endof-life care pathways where possible. To ensure generalisability, such trials should stratify participants according to different care settings including general acute care setting, emergency department, cancer care units, residential aged care facilities and specialist palliative care units. Additionally, if such studies demonstrate positive effects, it is imperative that there is careful ongoing evaluation of the implementation of any revised pathway as it is made available more broadly.

\section{Measurement (endpoints)}

In future studies, outcome measures should include the outcomes of interest in this review in relation to patients, families, carers and health professionals. These may include patients' symptom control, harms, communication between healthcare team and families, carers' well-being, grief and bereavement, staff and carers' satisfaction, staff confidence, cost of intervention, cost of care and medication use.

\section{Other}

Further, investigations of the effects of such pathways for specific populations are warranted. These specific populations may include, but are not limited to, children and patients with end-stage organ failure or dementia.

\section{ACKNOWLEDG MENTS}

The authors would like to acknowledge the contribution of the Cochrane Pain, Palliative and Supportive Care (PaPaS) Group and the Cochrane Effective Practice and Organisation of Care Group. In particular, we would like to thank Phil Wiffen, Professor Christopher Eccleston, Jessica Thomas, Joanne Abbott, Laila Tyrrell, Caroline Struthers and all the referees for their valuable input (peer reviewers for the original review: Bridget Candy and Alain Mayhew; consumer referees: Kathy Smith and Clare Jeffrey).

Cochrane Review Group funding acknowledgement: The National Institute for Health Research (NIHR) is the largest single funder of the Cochrane PaPaS Group. Disclaimer: The views and opinions expressed therein are those of the authors and do not necessarily reflect those of the NIHR, National Health Service (NHS) or the Department of Health. 


\section{R E F E R E N C E S}

\section{References to studies included in this review}

Costantini 2014 \{published data only\}

Costantini M, Romoli V, Di Leo S, Beccaro M, Bono L, Pilastri P, et al. Liverpool Care Pathway for patients with cancer in hospital: a cluster randomised trial. Lancet 2014;383:226-37.

\section{References to studies excluded from this review}

Bailey 2005 \{published data only\}

Bailey FA, Burgio KL, Woodby LL, Williams BR, Redden DT, Kovac $\mathrm{SH}$, et al. Improving processes of hospital care during the last hours of life. Archives of Internal Medicine 2005;165(15):1722-7.

\section{Bookbinder 2005 \{published data only\}}

Bookbinder M, Blank A, Arney E, Wollner D, Lesage P, McHugh M, et al. Improving end-of-life care: development and pilot-test of a clinical pathway. Journal of Pain and Symptom Management 2005;29(6):529-43.

\section{Chaplin 2009 \{published data only\}}

Chaplin D. Developing an end-of-life care pathway to improve nurses' bereavement care. Nursing Times 2009;105(1):20-1.

\section{Costantini 2013a \{published data only\}}

Costantini M, Pellergrini F, Di Leo S, Beccaro M, Rossi C, Glego G, et al. The Liverpool Care Pathway for cancer patients dying in hospital medical wards: a before-after cluster phase II trial of outcomes reported by family members. Palliative Medicine 2013 May 7 [Epub ahead of print].

\section{Costantini 2014a \{published data only\}}

Costantini M, Pellerini F, Di Leo S, Beccaro M, Rossi C, Flego G, et al. The Liverpool Care Pathway for cancer patients dying in hospital medical wards: a before-after cluster phase II trial of outcomes reported by family members. Palliative Medicine 2014;28(1):10-17.

\section{Di Leo 2013 \{published data only\}}

Di Leo S, Bono L, Romoli V, West E, Ambrosio R, Gallucci M, et al. Implementation of the Liverpool Care Pathway (LCP) for the dying patient in the inpatient hospice setting: development and preliminary assessment of the Italian LCP Program. American Journal of Hospice and Palliative Care 2014;31(1):61-8.

\section{Ellershaw 1997 \{published data only\}}

Ellershaw J, Murphy D, Shea T, Foster A, Overill S. Developing an integrated care pathway for the dying patient. European Journal of Palliative Care 1997;4:203-8.

\section{Ellershaw 2001 \{published data only\}}

Ellershaw J, Smith C, Overill S, Walker SE, Aldridge J. Care of the dying: setting standards for symptom control in the last 48 hours of life. Journal of Pain and Symptom Management 2001;21(1):12-7.
Ellershaw 2003 \{published data only\}

Ellershaw J, Ward C. Care of the dying patient: the last hours or days of life. BMJ 2003;326(7379):30-4.

\section{Ellershaw 2007 \{published data only\}}

Ellershaw J. Care of the dying: what a difference an LCP makes!. Palliative Medicine 2007;21(5):365-8.

\section{Fowell 2002 \{published data only\}}

Fowell A, Finlay I, Johnstone R, Minto L. An integrated care pathway for the last two days of life: Wales-wide benchmarking in palliative care. International Journal of Palliative Nursing 2002;8(12):566-73.

\section{Fowell 2003 \{published data only\}}

Fowell A, Finlay I, Johnstone R, Minto L. An integrated care pathway for the last two days of life: a pathway for implementing a pathway. Journal of Integrated Care Pathways 2003;7(3):91-9.

\section{Hardy 2007 \{published data only\}}

Hardy JR, Haberecht J, Maresco-Pennisi D, Yates P. Audit of the care of the dying in a network of hospitals and institutions in Queensland. Internal Medicine Journal 2007;37(5):315-9.

\section{Hockley 2005 \{published data only\}}

Hockley J, Dewar B, Watson J. Promoting end-of-life care in nursing homes using an 'integrated care pathway for the last days of life'. Journal of Research in Nursing 2005;10(2):135-52.

\section{Horey 2012 \{published data only\}}

Horey D, Street A, Sands A. Acceptability and feasibility of end-of-life care pathways in Australian residential aged care facilities. Medical Journal of Australia 2012;197(2):106-9.

\section{Jack 2003 \{published data only\}}

Jack BA, Gambles M, Murphy D, Ellershaw JE. Nurses' perceptions of the Liverpool Care Pathway for the dying patient in the acute hospital setting. International Journal of Palliative Nursing 2003;9(9):375-81.

\section{Johnson 2004 \{published data only\}}

Johnson DC, Kassner CT, Kutner JS. Current use of guidelines, protocols, and care pathways for symptom management in hospice. The American Journal of Hospice \& Palliative care 2004;21(1):51-7.

\section{Luhrs 2005 \{published data only\}}

Luhrs CA, Meghani S, Homel P, Drayton M, O'Toole E, Paccione $\mathrm{M}$, et al. Pilot of a pathway to improve the care of imminently dying oncology inpatients in a Veterans Affairs Medical Center. Journal of Pain and Symptom Management 2005;29(6):544-51.

\section{Main 2006 \{published data only\}}

Main J, Whittle C, Treml J, Woolley J, Main A. The development of an Integrated Care Pathway for all patients with advanced life-limiting illness - the Supportive Care Pathway. Journal of Nursing Management 2006;14(7):521-8. 
Matthews 2006 \{published data only\}

Matthews K, Gambles M, Ellershaw JE, Brook L, Williams M, Hodgson A, et al. Developing the Liverpool Care Pathway for the dying child. Paediatric Nursing 2006;18(1):18-21.

\section{Mellor 2004 \{published data only\}}

Mellor F, Foley T, Connolly M, Mercer V, Spanswick M. Role of a clinical facilitator in introducing an integrated care pathway for the care of the dying. International Journal of Palliative Nursing 2004;10(10):497-501.

\section{Mirando 2005 \{published data only\}}

Mirando S, Davies PD, Lipp A. Introducing an integrated care pathway for the last days of life. Palliative Medicine 2005;19(1):33-9.

\section{Neo 2012 \{published data only\}}

Neo PS, Poon MC, Peh TY, Ong SY, Koo WH, Santoso U, et al. Improvements in end-of-life care with a protocolbased pathway for cancer patients dying in a Singapore hospital. Annals of the Academy of Medicine Singapore 2012;41(11):489-93.

\section{Okon 2004 \{published data only\}}

Okon TR, Evans JM, Gomez CF, Blackhall LJ. Palliative educational outcome with implementation of PEACE tool integrated clinical pathway. Journal of Palliative Medicine 2004;7(2):279-95.

\section{Osterlind 2008 \{published data only\}}

Osterlind J, Hansebo G, Lantz G, Ternestedt BM. Pathways in end-of-life care for older people: care managers' reasoning. International Journal of Palliative Nursing 2008;14(9):420-5.

\section{Peterson 2000 \{published data only\}}

Peterson JL, Hartman HW. A pathway for patients who die within a week of hospice admission. International Journal of Palliative Nursing 2000;6(1):39-42.

Pooler 2003 \{published data only\}

Pooler J, McCrory F, Steadman Y, Westwell H, Peers S. Dying at home: a care pathway for the last days of life in a community setting. International Journal of Palliative Nursing 2003;9(6):258-64.

\section{Rose 2006 \{published data only\}}

Rose V. Evidence Base End of Life Care for Dying Patients and their Families in the Acute Health Care Setting. North Coast Area Health Service, NSW Health 2006.

\section{Taylor 2007 \{published data only\}}

Taylor AJ, Randall C. Process mapping: enhancing the implementation of the Liverpool Care Pathway. International Journal of Palliative Nursing 2007;13(4):163-7.

\section{Thompson-Hill 2009 \{published data only\}}

Thompson-Hill J, Hookey C, Salt E, O'Neill T. The supportive care plan: a tool to improve communication in end-of-life care. International Journal of Palliative Nursing 2009;15(5):250-5.
Veerbeek 2006 \{published data only\}

Veerbeek L, van Zuylen L, Gambles M, Swart SJ, van der Heide A, van der Rijt CC, et al. Audit of the Liverpool Care Pathway for the dying patient in a Dutch cancer hospital. Journal of Palliative Care 2006;22(4):305-8.

\section{Veerbeek 2008 \{published data only\}}

Veerbeek L, van der Heide A, de Vogel-Voogt E, de Bakker R, van der Rijt CC, Swart SJ, et al. Using the LCP: bereaved relatives' assessments of communication and bereavement. The American Journal of Hospice \& Palliative Care 2008;25(3):207-14.

Veerbeek 2008a \{published data only\} Veerbeek L, van Zuylen L, Swart SJ, van der Maas PJ, de VogelVoogt E, van der Rijt CC, et al. The effect of the Liverpool Care Pathway for the dying: a multi-centre study. Palliative Medicine 2008;22(2):145-51.

\section{Verhofstede 2014 \{published data only\}}

Verhofstede R, Smets T, Cohen J, Costantini M, Van Den Noortgate N, Van Der Heide A, et al. Development of the care programme for the last days of life for older patients in acute geriatric hospital wards: a phase $0-1$ study according to the Medical Research Council Framework. BMC Palliative Care 2015;14:24.

\section{References to ongoing studies}

Verhofstede 2014a \{published data only\}

Verhofstede R, Smets T, Cohen J, Costantini M, Van Den Noortgate N, Deliens L. Improving end-of-life care in acute geriatric hospital wards using the Care Programme for the Last Days of Life: study protocol for a phase 3 cluster randomized controlled trial. BMC Geriatrics 2015;15:13.

\section{Additional references}

\section{Addington-Hall 2007}

Addington-Hall JM. Introduction. Research Methods in Palliative Care. New York: Oxford University Press, 2007.

\section{Badger 2007}

Badger F, Thomas K, Clifford C. Raising standards for elderly people dying in care homes. European Journal of Palliative Care 2007;14(6):238-41

\section{Bruera 1991}

Bruera E, Kuehn N, Miller M, Selmser P, Macmillan K. The Edmonton Symptom Assessment System (ESAS): a simple method for the assessment of palliative care patients. Journal of Palliative Care 1991;7:6-9.

\section{Campbell 1998}

Campbell H, Hotchikiss R, Bradshaw N, Proteous M. Integrated care pathways. BMJ 1998;316:133-7.

\section{Centers for Disease Control and Prevention 2016}

Centers for Disease Control and Prevention. International Classification of Diseases,Ninth Revision (ICD-9). http:// www.cdc.gov/nchs/icd/icd9.htm 26 Jan 2016. 


\section{Chalmers 1983}

Chalmers TC, Celano P, Sacks HS, Smith H. Bias in treatment assignment in controlled clinical trials. New England Journal of Medicine 1983;309:1358-61.

\section{Chan 2014}

Chan R, Webster J, Phillips J, Currow D. The withdrawal of the Liverpool Care Pathway in the United Kingdom: what are the implications for Australia?. Medical Journal of Australia 2014;200(10):573.

\section{Chan 2014b}

Chan R. Towards better end-of-life care : a major opportunity for nurses to contribute to the debate. International Journal of Nursing Studies 2014;51(3):355-6.

\section{Cohen 1995}

Cohen S, Mount B, Strobel M, Bui F. The McGill Quality of Life Questionnaire: a measure of quality of life appropriate for people with advanced disease. A preliminary study of validity and acceptability. Palliative Medicine 1995;9(3):207-19.

\section{Costantini 2005}

Costantini M, Beccaro M, Merlo F. The last three months of life of Italian cancer patient. Methods, sample characteristics and response rate of the Italian survey of the dying of cancer (ISDOC). Palliative Medicine 2005;19:628-38.

\section{Currow 2011}

Currow, D. Evidence-based policy. Journal of Palliative Medicine 2011;14(7):803.

\section{Currow 2014}

Currow D, Abernethy A. Lessons from the Liverpool Care Pathway-evidence is key. The Lancet 2014;383(9913):192-3.

\section{Deeks 2003}

Deeks JJ, Dinnes J, D'Amico R, Sowden AJ, Sakaovitch C, Song F, et al. Evaluating non-randomised intervention studies. Health Technology Assessment 2003;7(27):1-4.

\section{Delvin 2009}

Delvin K. Controversial 'death' pathway. The Daily Telegraph 13 October 2009.

\section{Department of Health 2008}

Department of Health. End of life care strategy: promoting high quality care for all adults at the end of life. London: Department of Health, 2008.

\section{Department of Health and Ageing 2011}

Department of Health and Ageing. Supporting Australians to Live Well at the End of Life-National Palliative Care Strategy 2010. Canberra, Australia: Department of Health and Ageing, 2011.

\section{Di Leo 2015}

Di Leo S, Romoli V, Higginson I, Bulli F, Fantini S, Sguazzotti E, et al. 'Less ticking the boxes, more providing support': A qualitative study on health professionals' concerns towards the Liverpool Care of the Dying Pathway. Pallitative Medicine 2015;29(6):529-37.

\section{Ellershaw 2003a}

Ellershaw J, Wilkinson S. Care for the Dying: a Pathway to Excellence. Oxford: Oxford University Press, 2003.

\section{EPOC 2002}

Effective Practice and Organisation of Care Review Group. Data collection checklist, 2002. epoc.cochrane.org/sites/ epoc.cochrane.org/files/uploads/datacollectionchecklist.pdf (accessed 12 November 2013).

\section{Fowell 2004}

Fowell A, Russell I, Johnstone R, Finlay I, Russell D. Cluster randomisation or randomised consent as an appropriate methodology for trials in palliative care: a feasibility study [ISRCTN60243484]. BMC Palliative Care 2004;3(1):1.

\section{Higgins 2011}

Higgins JPT, Green S (editors). Cochrane Handbook for Systematic Reviews of Interventions Version 5.1.0 [updated March 2012]. The Cochrane Collaboration, 2011. Available from www.cochrane-handbook.org.

\section{Karlawish 2003}

Karlawish JH. Conducting research that involves subjects at the end of life who are unable to give consent. Journal of Pain and Symptom Management 2003;25(4):S14-24.

\section{Kawn 2004}

Kawn J, Sandercock, Pater AG. In-hospital care pathways for stroke. Cochrane Database of Systematic Reviews 2004, Issue 4. [DOI: 10.1002/14651858.CD002924.pub2]

\section{Lemmens 2008}

Lemmens L, van Zelm R, Vanhaecht K, Kerkkamp H. Systematic review: indicators to evaluate effectiveness of clinical pathways for gastrointestinal surgery. Journal of Evaluation in Clinical Practice 2008;14(5):880-7.

\section{Lunney 2003}

Lunney JR, Lynn J, Foley DJ, Lipson S, Guralnik JM. Patterns of functional decline at the end of life. JAMA 2003;2879(19):2387-92.

\section{McConnell 2013}

McConnell T, O'Halloran P, Porter S, Donnelly M. Systematic realist review of key factors affecting the successful implementation and sustainability of the Liverpool Care Pathway for the dying patient. Worldviews on Evidence-Based Nursing 2013;10(4):218-37.

\section{Medical Research Council 2000}

Medical Research Council. A framework for development and evaluation of RCTs for complex interventions to improve health. Medical Research Council, 2000.

\section{Mills 1994}

Mills M, Davies H, Macrae W. Care of dying patients in hospital. BMJ 1994;309:583-6. 


\section{Ministry of Health NZ 2013}

Ministry of Health NZ. Resource and Capability Framework for Integrated Adult Palliative Care Services in New Zealand. Wellington: Ministry of Health NZ, 2013.

\section{Mularski 2007}

Mularski R, Dy S, Shugarman S, Wilkinson A, Lynn J, Shekelle P, et al. A systematic review of measures of end-of-life care and its outcomes. Health Services Research 2007;42(5):1848-70.

\section{Neuberger 2013}

Neuberger J, Guthrie C, Aaronvitch D, Hameed K, Bonser T, Harries R, et al. More care, less pathway: a review of the Liverpool Care Pathway, 2013. www.gov.uk/government/ uploads/system/uploads/attachment_data/file/212450/ Liverpool_Care_Pathway.pdf.

\section{Parry 2013}

Parry R, Seymour J, Whittaker B, Bird L, Cox K. Rapid evidence review: pathways focused on the dying phase in end of life care and their key components. National End of Life Care Programme, NHS, 2013.

\section{Phillips 2011}

Phillips J, Halcomb E, Davidson P. End-of-life care pathways in acute and hospice care: an integrative review. Journal of Pain and Symptom Management 2011;41(5):940-55.

\section{Portenoy 1994}

Portenoy R, Thaler H, Kornblith A, McCarthy L, Friedlanderklar $\mathrm{H}$, Sobel K, et al. The Memorial Symptom Assessment Scale: an instrument for the evaluation of symptom prevalence, characteristics and distress. European Journal of Cancer 1994;30(9):1326-36.

\section{RevMan 2014 [Computer program]}

The Nordic Cochrane Centre, The Cochrane Collaboration. Review Manager (RevMan). Version 5.3. Copenhagen: The Nordic Cochrane Centre, The Cochrane Collaboration, 2014.

\section{Reyna 2008}

Reyna Y, Bennett M, Bruera E. Ethical and practical issues in designing and conducting clinical trials in palliative care. In: Addington-Hall J, Bruera E, Higginson I, Payne S editor(s). Research Methods in Palliative Care. Oxford: Oxford University Press, 2008.

\section{Rotter 2008}

Rotter T, Kugler J, Koch R, Gothe H, Twork S, van Oostrum JM, et al. A systematic review and meta-analysis of the effects of clinical pathways on length of stay, hospital costs, and patient outcomes. BMC Health Service Research 2008;8(1):265.

\section{Sacks 1982}

Sacks H, Chalmers TC, Smith JH. Randomized versus historical controls for clinical trials. American Journal of Medicine 1982;72:233-40.

\section{Schultz 2010}

Schultz KF, Altman DG, Moher D, Consort Group. Consort 2010 statement: Updated guidelines for reporting parallel group randomised trials. PLoS Medicine 2010;7(3):e1000251.

\section{Smith 2009}

Smith R. More training needed following concerns about death pathway: cancer tsar. The Daily Telegraph 17 October 2009.

\section{Steinhauser 2000}

Steinhauser KE, Christakis NA, Clipp EC, McNeilly M, McIntyre L, Tulsky JA. Factors considered important at the end of life by patients, family, physicians and other care providers. JAMA 2000;284(19):2476-82.

\section{Steinhauser 2001}

Steinhauser KE, Christakis NA, Clipp EC, McNeilly M, Grambow S, Parker J, et al. Preparing for the end of life: preferences of patients, families, physicians, and other care providers. Journal of Pain and Symptom Management 2001;22(3):727-37.

\section{Teno 2001}

Teno JM, Clarridge B, Casey V, Edgman-Levitan S, Fowler J. Validation of Toolkit After-Death Bereaved Family Member Interview. Journal of Pain and Symptom Management 2001;22(3):752-8.

\section{Travis 2002}

Travis S, Bernard M, Dixon S, McAuley W, Loving G, McClanahan L. Obstacles to palliation and end-of-life care in a long-term care facility. The Gerontologist 2002;42(3):342-9.

\section{United Nations 2013}

United Nations. World Population Ageing 2013. United Nations, Department of Economic and Social Affairs, Population Division, 2013.

\section{WHO 2009}

World Health Organization. WHO definition of palliative care. www.who.int/cancer/palliative/definition/en/ (accessed 12 November 2013).

\section{WHO 2011}

World Health Organization. Palliative Care for Older People: better practices. Geneva: WHO, 2011.

\section{WHO and Cancer Research UK 2012}

World Health Organization and Cancer Research UK. World Cancer Factsheet. WHO and Cancer Research UK, 2012.

\section{Zigmond 1983}

Zigmond A, Snaith R. The Hospital Anxiety and Depression Scale. Acta Psychiatrica Scandinavica 1983;67(6):361-70.

\section{References to other published versions of this review Chan 2010}

Chan R, Webster J. End-of-life care pathways for improving outcomes in caring for the dying. Cochrane 
Database of Systematic Reviews 2010, Issue 1. [DOI: 10.1002/14651858.CD008006.pub2]

\section{CHARACTERISTICS OF STUDIES}

Characteristics of included studies [ordered by study ID]

\section{Chan 2013}

Chan R, Webster J. End-of-life care pathways for improving outcomes in caring for the dying. Cochrane Database of Systematic Reviews 2013, Issue 11. [DOI: 10.1002/14651858.CD008006.pub3]

Costantini 2014

Methods

Objective: To assess the effectiveness of LCP in the Italian context (LCP-I) in improving the quality of end-of-life care for patients with cancer in hospitals and for their family

Study design: Pragmatic cluster randomised trial

Object of randomisation: Wards stratified by region and matched for assessment period

\section{Recruitment:}

Ward Level

- No information was provided

Individual Level

- Information about the patient, closest family member during the last week of the patients' life in hospital, and the general practitioner was obtained for all cancer deaths. Two months after the patients' death, the regional co-ordinator sent a letter to the identified family member to introduce the study. A subsequent telephone contact was made to ascertain agreement for participation

Allocation: Randomly assigned

Total number approached: 16 (15 general hospitals and one university hospital)

Number recruited: 16 wards (8 pairs of wards). Family members interviewed: 119 intervention arm, 113 standard care

\section{Method of analysis:}

- Charateristics of patients and centres were reported as mean and standard deviation or frequency and percentage respectively for continuous and categorical variables

- Distribution of variables for control and intervention wards was compared using the t-Student test or one way ANOVA

- Binary or nominal variables were compared using the Pearson Chi-square test

- The assessment of the primary end-point took into account the clustered design of the trial through the use of hierarchical linear models. These models were adjusted for the average level of quality of care provided to the baseline assessment. The results were expressed in terms of beta coefficients of regression or in estimated marginal means, $95 \%$ confidence intervals $(95 \% \mathrm{Cl})$ and level of significance. $\mathrm{P}<0.05$ was considered statistically significant. The same approach was used for the assessment of the continuous secondary end-points where there were continual variables. For the assessment of categorical secondary end-points with categorical variables, the hierarchical logistical model was used

Follow-up: 2-4 months after the patients' death or a telephone interview (up to 1 year) as a second choice

Participants

Country: Italy

Clinical setting: 16 general medicine wards

Inclusion criteria: 
Ward Level

- classified in the regional records as 'Medical', 'General Medical' or 'Internal Medical' ward

- at least 25 cancer deaths on the ward per year

- consent from the hospital management to participate in the trial

- consent from the head of the medical ward to participate in the trial

- consent from the hospital management and the head of the ward, and a specialist palliative care team (from inside or outside the hospital) to implement the LCP-I programme in the ward.

Individual Level

- A total of 308 patients who died from cancer (intervention $=147$; control $=161$ ) were enrolled. A total of 232 family members (intervention $=119$; control $=113$ ) were interviewed

\section{Exclusion criteria:}

Ward Level

- If in the same hospital another medical ward has already been randomly selected to participate in the research programme (regardless of which arm was randomised)

Individual Level

- Those who were relatives of a doctor or a nurse working in the hospital

Age: Mean age of patients: intervention = 75.6 (SD 10.8); Control 75.2 (SD 11.9) - Mean age of family members: Intervention 54.2 (SD 11.4); Control 55.6 (SD 11.2)

Gender: Patient 190 male/118 female, family member: 107 male/201 female

Primary cancer site: Gastrointestinal: 84; Respiratory: 75; Genitourinary: 34; Haematological: 59; Breast: 23; Other: 33

Ethnicity: Not stated

Interventions

Intervention arm: The LCP-I programme: a continuous quality improvement programme of end-oflife care implemented by a PCU in a hospital medical ward. The LCP-I is a complex 10-step intervention which involves: development of the project in the targeted setting (three steps); implementing of the project over a 6-month period (five steps) and development of a sustainability strategy (two steps)

Control arm: Usual care without the LCP-I programme

Outcomes Primary outcomes:

Overall quality of care assessed using the "Overall rating of patient focused, family-centred care toolkit scale"

\section{Secondary outcomes:}

Respect, dignity, and kindness scale

Advance care planning

Overall control of pain, breathlessness, and nausea and vomiting

Notes Trial registration: ClinicalTrials.gov Identifier: NCT01081899

\section{Risk of bias}

\begin{tabular}{lll}
\hline Bias & Authors' judgement & Support for judgement \\
\hline $\begin{array}{l}\text { Random sequence genera- } \\
\text { tion (selection bias) }\end{array}$ & Low risk & $\begin{array}{l}\text { Randomisation was centralised at the trial centre of the National Cancer Re- } \\
\text { search Institute of Genoa, which verified the eligibility and recorded details of }\end{array}$ \\
\hline
\end{tabular}


Costantini 2014 (Continued)

each pair of wards and matched palliative care teams, assigned a numerical code for identification, and recorded the allocation

\begin{tabular}{lll}
\hline $\begin{array}{l}\text { Allocation concealment } \\
\text { (selection bias) }\end{array}$ & Unclear risk & No information was found \\
\hline $\begin{array}{l}\text { Blinding of participants } \\
\text { and personnel (perfor- } \\
\text { mance bias) } \\
\text { All outcomes }\end{array}$ & High risk & $\begin{array}{l}\text { Due to the nature of the intervention, the hospital staff, PCTs, and interviewers } \\
\text { could not be masked to the allocation status. Family members were informed } \\
\text { about the general aim of the study but not of the group assignment. However, } \\
\text { it is likely that family members would have been aware of the intervention }\end{array}$ \\
\hline $\begin{array}{l}\text { Blinding of outcome as- } \\
\text { sessment (detection bias) } \\
\text { All outcomes }\end{array}$ & High risk & $\begin{array}{l}\text { Due to the nature of the intervention, the hospital staff, PCTs, and interviewers } \\
\text { could not be masked to the allocation status. Family members were informed } \\
\text { about the general aim of the study but not of the group assignment. However, } \\
\text { it is unlikely to mask the family members from the intervention }\end{array}$
\end{tabular}

Incomplete outcome data High risk (attrition bias)

All outcomes

$232(75 \%)$ of 308 family members were interviewed, 119 (81\%) of 147 with relatives cared for in the LCP-I wards (mean cluster size 14.9, range: 8-22) and 113 (70\%) of 161 in the control wards (14.1, range: 8-22)

Attrition and exclusions were reported for each group and reasons for attrition described. However, there was an imbalance in attrition between groups, which may have affected outcomes. Similarly, there was a difference in reasons for attrition between groups

\begin{tabular}{|c|c|c|}
\hline $\begin{array}{l}\text { Selective reporting (re- } \\
\text { porting bias) }\end{array}$ & Unclear risk & $\begin{array}{l}\text { The authors appeared to have reported all that they planned to measure. } \\
\text { However, trial registration "first received: March 4, 2010" by ClinicalTrials.gov, } \\
\text { which was four months after the trial commenced }\end{array}$ \\
\hline
\end{tabular}

Other bias Unclear risk Only $34 \%$ of patients were cared for in accordance with the programme

LCP: Liverpool Care Pathway

LCP-I: Italian version of the Liverpool Care Pathway

PCT: palliative care team

Characteristics of excluded studies [ordered by study ID]

\begin{tabular}{ll}
\hline Study & Reason for exclusion \\
\hline Bailey 2005 & Before-and-after study (without control) \\
\hline Bookbinder 2005 & $\begin{array}{l}\text { Controlled before-and-after study: non-contemporaneous data collection, non-comparable sam- } \\
\text { pling }\end{array}$ \\
\hline Chaplin 2009 & Non-experimental study: case report \\
\hline Costantini 2013a & Before-and-after study (without control) \\
\hline Costantini 2014a & Before-and-after study (without control) \\
\hline Di Leo 2013 & Non-experimental study: implementation phase 0-1 study (without control) \\
\hline Ellershaw 1997 & Non-experimental study: case report \\
\hline Ellershaw 2001 & Non-experimental study: audit \\
\hline
\end{tabular}




\begin{tabular}{|c|c|}
\hline Study & Reason for exclusion \\
\hline Ellershaw 2003 & Review \\
\hline Ellershaw 2007 & Letter \\
\hline Fowell 2002 & Non-experimental study: audit \\
\hline Fowell 2003 & Non-experimental study: audit \\
\hline Hardy 2007 & Non-experimental study: audit \\
\hline Hockley 2005 & Non-experimental study: qualitative study \\
\hline Horey 2012 & Non-experimental study \\
\hline Jack 2003 & Non-experimental study: qualitative study/focus groups \\
\hline Johnson 2004 & Non-experimental study: cross-sectional survey study \\
\hline Luhrs 2005 & $\begin{array}{l}\text { Controlled before-and-after study: non-contemporaneous data collection, non-comparable sam- } \\
\text { pling and does not have at least } 2 \text { intervention and } 2 \text { control sites }\end{array}$ \\
\hline Main 2006 & Non-experimental study: case report \\
\hline Matthews 2006 & Non-experimental study: audit \\
\hline Mellor 2004 & Qualitative study \\
\hline Mirando 2005 & Non-experimental study: audit \\
\hline Neo 2012 & Non-experimental study: retrospective audit \\
\hline Okon 2004 & Controlled before-and-after study: does not have at least 2 intervention and 2 control sites \\
\hline Osterlind 2008 & Non-experimental study: qualitative study \\
\hline Peterson 2000 & Non-experimental study: case report \\
\hline Pooler 2003 & Non-experimental study: audit \\
\hline Rose 2006 & Non-experimental study: audit \\
\hline Taylor 2007 & Before-and-after study (without control) \\
\hline Thompson-Hill 2009 & Before-and-after study (without control) \\
\hline Veerbeek 2006 & Non-experimental study: audit \\
\hline Veerbeek 2008 & Before-and-after study (without control) \\
\hline Veerbeek 2008a & Before-and-after study (without control) \\
\hline Verhofstede 2014 & Non-experimental study \\
\hline
\end{tabular}


Characteristics of ongoing studies [ordered by study ID]

Verhofstede 2014a

Trial name or title

Improving end-of-life care in acute geriatric hospital wards using the Care Programme for the Last Days of Life: study protocol for a phase 3 cluster randomised controlled trial

Methods

Objective: To evaluate the effects of the Care Programme for the Last Days of Life

Study design: Cluster randomised trial

Object of randomisation: Hospitals

\section{Recruitment:}

Hospital Level

- study presented at three geriatric meetings and information letters sent to geriatricians following meetings

Individual Level

- Not stated

Allocation: Randomly assigned

Total number approached: 10 hospitals

\section{Method of analysis:}

- Data cleaning will be performed via SPSS syntax operations

- all statistical tests will be done two-tailed with $95 \%$ confidence intervals. $\mathrm{P}<0.05$ will be considered statistically significant

Participants

\section{Country: Belgium}

Clinical Setting: 10 hospitals with one or more acute geriatric wards

\section{Inclusion Criteria:}

Hospital Level

- the cluster or hospital has one or more acute geriatric wards

- the medical and nursing head of one or more acute geriatric wards per hospital give consent for participation in the study

Individual Level

- those dying in the acute geriatric ward between October 2012 and March 2015

- those that have been hospitalised for more than 48 hours

- those having given informed consent at admission for the use of their personal information from medical or nursing records for the purposes for the study

\section{Exclusion Criteria:}

Not stated participating hospitals prior to randomisation; during which time care as usual will be provided.

The Care Programme will be implemented and subsequently established according to a nine component implementation guide:

1. establishing the implementation project and preparing the environment for organisational changes 
2. preparing the documentation

3. baseline review

4. training geriatric healthcare staff

5. use of the Care Guide for Last Days of Life with intensive support

6. semi-intensive support

7. evaluation

8. consolidation and

9. on-going education, training and support

Intervention arm: The Care Programme for the Last Days of Life will be implemented over a sixmonth period with the support of an implementation guide (as detailed above). A one-year postintervention assessment will be conducted following the implementation period

Control arm: Will continue to provide care as usual and will conduct a one-year postintervention assessment directly following the one-year baseline assessment

Outcomes

\section{Primary outcomes:}

Quality of dying during the last 48 hours of life: the patient's symptom frequency and symptom burden measured using the EOLD-SM and EOLD-CAD

\section{Secondary outcomes:}

- the quality of care during the last three days of life as perceived by nurses, i.e. physical symptoms, emotional, psychological and spiritual/existential needs and provision of information and support measured using the POS

- the quality of care during the last 48 hours of life as perceived by family carers, i.e. satisfaction with the care provided to the patient during the last 48 hours of life measured using the EOLD-SWC

- the content of care during the last 48 hours of life, i.e. the goal of treatment, medical and nursing interventions, medication policy

- the communication among clinical staff i.e. informing the family physician about the impending death

- the communication between clinical staff and patients and/or family carers i.e. the perception of communication with the physician during the dying phase by family carers measured using the FPPFC

- the level of bereavement of family carers after the death of the patient measured using the PGD scale

Starting date Sept 2012 (information from ClinicalTrials.gov)

Contact information Rebecca Verhofstede: rebecca.verhofstede@vub.ac.be End-of-Life Care Research Group, Vrije Universiteit Brussel (VBU) \& Ghent University, Brussels, Belgium

Published Protocol: Protocol received 19 December 2014, accepted 3 February 2015, published online 22 February 2015

POS - Palliative Care Outcome Scale

EOLD-SWC - End-of-Life in Dementia Scales Symptom Management

FPPFC - Family Perception of Physician-Family Caregiver Communication

PGD - Prolonged Grief Disorder 


\section{APPENDICES}

\section{Appendix 1. CENTRAL search strategy}

\#1 MeSH descriptor: [Palliative Care] this term only

\#2 palliat*

\#3 end-of-life

\#4 terminally ill

\#5 MeSH descriptor: [Terminally III] this term only

\#6 dying

\#7 hospice

\#8 MeSH descriptor: [Hospices] explode all trees

\#9 end-stage

$\# 10 \# 1$ or \#2 or \#3 or \#4 or \#5 or \#6 or \#7 or \#8 or \#9

\#11 MeSH descriptor: [Critical Pathways] this term only

\#12 ((clinical or critical or care) next path*)

\#13 (care next (map* or plan*))

\#14 MeSH descriptor: [Guideline] explode all trees

\#15 MeSH descriptor: [Health Planning Guidelines] this term only

\#16 MeSH descriptor: [Guideline Adherence] this term only

\#17 (compliance next (protocol ${ }^{\star}$ or policy or guideline $\left.{ }^{\star}\right)$ )

\#18 (guideline ${ }^{\star}$ near/2 (introduc ${ }^{\star}$ or issu* or impact or effect ${ }^{\star}$ or disseminat $^{\star}$ or distribut $^{\star}$ or implement $^{\star}$ ))

\#19 "nursing protocol*"

\#20 "professional standard*"

\#21 (practice guidelin* or practice protocol ${ }^{\star}$ or clinical practice guidelin*)

\#22 \#11 or \#12 or \#13 or \#14 or \#15 or \#16 or \#17 or \#18 or \#19 or \#20 or \#21

\#23 \#10 and \#22

\section{Appendix 2. MEDLINE and MEDLINE In-progress (Ovid) search strategy}

Database: Ovid MEDLINE(R)

1 Palliative Care/ or palliat\$.mp.

2 end-of-life.mp.

3 terminally ill.mp. or Terminally Ill/

4 dying.mp.

5 hospice.mp. or Hospices/

6 end-stage.mp. 
7 or/1-6

8 Critical Pathways/

9 ((clinical or critical or care) adj path\$).mp.

10 (care adj (map\$ or plan\$)).mp.

11 exp Guideline/

12 Health Planning Guidelines/

13 Guideline Adherence/

14 (compliance adj (protocol? or policy or guideline?)).mp.

15 (guideline? adj2 (introduc\$ or issu\$ or impact or effect? or disseminat\$ or distribut\$ or implement\$)).mp.

16 nursing protocol?.mp.

17 professional standard\$.mp.

18 (practice guidelin\$ or practice protocol\$ or clinical practice guidelin\$).mp.

19 or/8-18

20 Guideline.pt.

21 randomized controlled trial.pt.

22 controlled clinical trial.pt.

23 Intervention Studies/

24 experiment\$.mp.

25 (time adj series).mp.

26 (pre test or pretest or post test or posttest).mp.

27 Random Allocation/

28 impact.mp.

29 intervention?.mp.

30 Evaluation Studies/

31 Comparative Study.pt.

32 Humans/

33 or $/ 20-31$

347 and 19 and 32 and 33 (1096)

\section{Appendix 3. EMBASE (Ovid) search strategy}

1 Palliative Care/ or palliat\$.mp.

2 end-of-life.mp.

3 terminally ill.mp. or Terminally Ill/

4 dying.mp.

5 hospice.mp. or Hospices/

6 end-stage.mp. 
7 or/1-6

8 Critical Pathways/

9 ((clinical or critical or care) adj path\$).mp.

10 (care adj (map\$ or plan\$)).mp.

11 exp Practice Guideline/

12 Health Planning Guidelines/

13 Guideline Adherence/

14 (compliance adj (protocol? or policy or guideline?)).mp.

15 (guideline? adj2 (introduc\$ or issu\$ or impact or effect? or disseminat\$ or distribut\$ or implement\$)).mp.

16 nursing protocol?.mp.

17 professional standard\$.mp.

18 (practice guidelin\$ or practice protocol\$ or clinical practice guidelin\$).mp.

19 or/8-18

20 randomized controlled trial/

21 controlled clinical trial/

22 Intervention Studies/

23 experiment\$.mp.

24 (time adj series).mp.

25 (pre test or pretest or post test or posttest).mp.

26 Random Allocation/

27 impact.mp.

28 intervention?.mp.

29 Evaluation Studies/

30 comparative study/

31 Humans/

32 or/20-30

337 and 19 and 32 and 31

\section{Appendix 4. PsycINFO (Ovid) search strategy}

1 Palliative Care/ or palliat\$.mp.

2 end-of-life.mp.

3 terminally ill.mp. or Terminally Ill/

4 dying.mp.

5 hospice.mp. or Hospices/

6 end-stage.mp.

7 or/1-6

End-of-life care pathways for improving outcomes in caring for the dying (Review) 
8 ((clinical or critical or care) adj path\$).mp.

9 (care adj (map\$ or plan\$)).mp.

10 (compliance adj (protocol? or policy or guideline?)).mp.

11 (guideline? adj2 (introduc\$ or issu\$ or impact or effect? or disseminat\$ or distribut\$ or implement\$)).mp.

12 nursing protocol?.mp.

13 professional standard\$.mp.

14 (practice guidelin\$ or practice protocol\$ or clinical practice guidelin\$).mp.

15 (or/8-9) or (or/10-14)

167 and 15

\section{Appendix 5. CINAHL (EBSCO) search strategy}

S38 S23 AND S36 AND S37 Search modes - Boolean/Phrase Interface - EBSCOhost

S37 S24 OR S25 OR S26 OR S27 OR S28 OR S29 OR S30 OR S31 OR S32 OR S33 OR S34 OR S35 Search modes - Boolean/Phrase Interface - EBSCOhost

S36 (MH "Human") Search modes - Boolean/Phrase Interface - EBSCOhost

S35 PT comparative study Search modes - Boolean/Phrase Interface - EBSCOhost

S34 (MH "Evaluation Research") Search modes - Boolean/Phrase Interface - EBSCOhost

S33 intervention* Search modes - SmartText Searching Interface - EBSCOhost

S32 intervention* Search modes - Boolean/Phrase Interface - EBSCOhost

S31 impact Search modes - Boolean/Phrase Interface - EBSCOhost

S30 (MH "Random Assignment") Search modes - Boolean/Phrase Interface - EBSCOhost

S29 (pre test or pretest or post test or posttest) Search modes - Boolean/Phrase Interface - EBSCOhost

S28 "time series" Search modes - Boolean/Phrase Interface - EBSCOhost

S27 experiment* Search modes - Boolean/Phrase Interface - EBSCOhost

S26 (MH "Experimental Studies") Search modes - Boolean/Phrase Interface - EBSCOhost

S25 PT randomized controlled trial Search modes - Boolean/Phrase Interface - EBSCOhost

S24 PT guideline Search modes - Boolean/Phrase Interface - EBSCOhost

S23 S22 Limiters - Published Date from: 20090801-20130131

Search modes - Boolean/Phrase Interface - EBSCOhost

S22 (S10 AND S21) Search modes - Boolean/Phrase Interface - EBSCOhost

S21 S11 OR S12 OR S13 OR S14 OR S15 OR S16 OR S17 OR S18 OR S19 OR S20 Search modes - Boolean/Phrase Interface - EBSCOhost S20 ("practice guidelin*" or "practice protocol*" or "clinical practice guidelin*") Search modes - Boolean/Phrase Interface - EBSCOhost S19 "professional standard\$\$" Search modes - Boolean/Phrase Interface - EBSCOhost

S18 "nursing protocol?" Search modes - Boolean/Phrase Interface - EBSCOhost

S17 (guideline? N2 (introduc* or issu* or impact or effect? or disseminat* or distribut* or implement ${ }^{\star}$ )) Search modes - Boolean/Phrase Interface - EBSCOhost

S16 (compliance N1 (protocol? or policy or guideline?)) Search modes - Boolean/Phrase Interface - EBSCOhost 
S15 (MH "Guideline Adherence") Search modes - Boolean/Phrase Interface - EBSCOhost

S14 (MH "Practice Guidelines") Search modes - Boolean/Phrase Interface - EBSCOhost

S13 "care map*" or "care plan*" Search modes - Boolean/Phrase Interface - EBSCOhost

S12 "clinical path" or "critical path" or "care path*" Search modes - Boolean/Phrase Interface - EBSCOhost

S11 (MH "Critical Path") Search modes - Boolean/Phrase Interface - EBSCOhost

S10 S1 OR S2 OR S3 OR S4 OR S5 OR S6 OR S7 OR S8 OR S9 Search modes - Boolean/Phrase Interface - EBSCOhost

S9 end-stage Search modes - Boolean/Phrase Interface - EBSCOhost

S8 (MH "Hospices") Search modes - Boolean/Phrase Interface - EBSCOhost

S7 hospice Search modes - Boolean/Phrase Interface - EBSCOhost

S6 dying Search modes - Boolean/Phrase Interface - EBSCOhost

S5 (MH "Terminally Ill Patients") Search modes - Boolean/Phrase Interface - EBSCOhost

S4 "terminally ill" Search modes - Boolean/Phrase Interface - EBSCOhost

S3 end-of-life Search modes - Boolean/Phrase Interface - EBSCOhost

S2 Palliative Care Search modes - Boolean/Phrase Interface - EBSCOhost

S1 (MH "Palliative Care") Search modes - Boolean/Phrase Interface - EBSCOhost

WHAT'S NEW

\begin{tabular}{lll}
\hline Date & Event & Description \\
\hline 13 November 2018 & Review declared as stable & See Published notes. \\
\hline
\end{tabular}

\section{H I S T O R Y}

Protocol first published: Issue 4, 2009

Review first published: Issue 1, 2010

\begin{tabular}{lll}
\hline Date & Event & Description \\
\hline 18 August 2017 & Review declared as stable & See Published notes. \\
\hline 1 July 2015 & $\begin{array}{l}\text { New citation required but conclusions } \\
\text { have not changed }\end{array}$ & $\begin{array}{l}\text { The review has been amended to include one new study meeting } \\
\text { the inclusion criteria; we identified one ongoing study; the con- } \\
\text { clusions remained unchanged. }\end{array}$ \\
\hline 27 November 2013 & New search has been performed & $\begin{array}{l}\text { This review has been updated to include the results of a new } \\
\text { search and a new 'Risk of bias' table. }\end{array}$ \\
\hline 23 August 2013 & New search has been performed & $\begin{array}{l}\text { A new search was run in June 2013, but no new studies met crite- } \\
\text { ria for inclusion in the review update. }\end{array}$ \\
\hline 15 August 2013 & $\begin{array}{l}\text { New citation required but conclusions } \\
\text { have not changed }\end{array}$ & \begin{tabular}{l} 
New Citation: Conclusion not changed. \\
\hline
\end{tabular}
\end{tabular}




\section{CONTRIBUTIONS OF AUTHORS}

Writing the protocol: RC, JW.

Developing the search strategy: RC and Joanne Abbott, the Trials Search Co-ordinator, Cochrane PaPaS Group.

Searching for trials: RC, JW, AB.

Selecting trials: RC, JW.

Data entry: RC, JW, JB.

Analysis: RC, JW, AB.

Interpreting analysis: RC, JW, $A B$.

Drafting final review: $R C, J W, A B$.

Updating the review: $R C, J W, A B$.

\section{DECLARATIONS OF INTEREST}

RC has no relevant conflict of interest to declare.

JW has no relevant conflict of interest to declare.

$A B$ has no relevant conflict of interest to declare.

\section{SOURCES OF SUPPORT}

\section{Internal sources}

- Royal Brisbane and Women's Hospital, Metro North Hospital and Health Service, Queensland University of Technology, and National Health and Medical Research Council, Australia.

For funding the salary and facilities for RC to conduct this systematic review

- Royal Brisbane and Women's Hospital, Metro North Hospital and Health Service, Brisbane, Australia.

For funding the salary and facilities for JW to conduct this systematic review

- West Moreton Hospital and Health Services, Australia.

For funding the salary and facilities for $A B$ to conduct this systematic review

\section{External sources}

- No sources of support supplied

\section{DIFFERENCES BETWEEN PROTOCOL AND REVIEW}

Under 'Measures of treatment effect', the protocol stated that risk ratio (RR) with their $95 \%$ confidence intervals (Cls) would be used. We were unable to include the RR, as the only included trial reported the outcomes using odds ratio (OR) with $95 \% \mathrm{Cls}$. In this instance, we did not request raw data from the trialists to undertake further analysis.

We added adverse effects to the primary outcomes in line with current Cochrane standards, and spiritual needs to the secondary outcomes.

\section{NOTES}

\section{August 2017}

A restricted search in August 2017 did not identify any potentially relevant studies likely to change the conclusions. Therefore, this review has now been stabilised following discussion with the authors and editors. We will reassess this review for updating in 2018. If appropriate, we will update the review before this date if new evidence likely to change the conclusions is published, or if standards change substantially which necessitate major revisions.

\section{November 2018}

At November 2018, the authors and editors are not aware of any potentially eligible new studies. The Liverpool Care Pathway has been phased out in the UK. Other countries that are continuing with the practice tend to change the name of the approach to "end-of-life care plans". We do not think it is likely that large, high quality trials with the potential to change the direction of the conclusions will be published. We will reassess this review for updating in five years. If appropriate, we will update the review before this date if new evidence likely to change the conclusions is published, or if standards change substantially which necessitate major revisions. 


\section{N D EX TERMS}

\section{Medical Subject Headings (MeSH)}

${ }^{\star}$ Critical Pathways; *Terminal Care [psychology]; Dyspnea [prevention \& control]; Nausea [prevention \& control]; Pain Management; Quality of Life; Randomized Controlled Trials as Topic; Treatment Outcome; Vomiting [prevention \& control]

\section{MeSH check words}

Humans 